\title{
Detecting essential and removable interactions in genome-wide association studies
}

\author{
Chengqing Wu*, Hong Zhang*, Xiangtao Liu, Andrew DeWan, \\ Robert Dubrow, Zhiliang Ying, Yaning Yang, \\ AND JOSEPHINE $\mathrm{HOH}^{\dagger}$
}

Detection of disease gene interaction effects among the enormous array of single nucleotide polymorphism (SNP) combinations represents the next frontier in genome-wide association (GWA) studies. Here we propose a novel strategy on the basis of the pattern and nature of the interaction, which can be classified as essential (EI) or removable (RI). We provide an analytical framework, including the qualitative conditions for screening EIs/RIs and a RI-to-EI likelihood ratio score to quantitatively measure the effect. In analyzing six GWA data sets, we find that the scores follow an exponential distribution, except in the upper $10^{-8}$ tail region in which the scores become irregular and unpredictable. Our approach is conceptually simple, computationally efficient and detects interactions that can be visualized and unequivocally interpreted.

AMS 2000 SUBJECt ClASSIFICATIONS: Primary 62P10, 62F03; secondary 92D10.

KEYWORDS AND PHRASES: Genome-wide association study, gene-gene interaction, removable interaction, essential interaction, likelihood ratio statistics.

\section{INTRODUCTION}

A growing number of successful genome-wide association (GWA) studies have been reported in the past several years [6]. All have initially focused on the main effects of individual single nucleotide polymorphisms (SNPs), which for the most part have been found to be relatively weak. Yet to be explored in this wealth of data are the interaction effects among SNPs, wherein the main effect of one SNP may be weak because that SNP confers risk only in the presence of one or more other SNPs. Taking these gene-gene interactions into account may lead to the discovery of stronger and more interesting effects [2, 8]. However, searching for meaningful interactions is a challenge for even the simplest case of examining all two-way interactions among a million SNPs ( half of $10^{12}$ combinations).

\footnotetext{
*Equal contributions.

${ }^{\dagger}$ Corresponding author.
}

To aid in efficiency and interpretability of this formidable computational problem, we present an analytical framework for two types of interactions, removable interactions (RIs) and essential interactions $[1,12]$. Interaction is usually defined as departure from additivity of effects on a specific outcome scale. If a monotone transformation exists that induces additivity, the interaction is called removable; otherwise, the interaction is essential.

What makes the mathematics hard is the sheer volume of networks in GWA data, for which no universal law underlying the system can be deduced. Our idea to detect interactions in GWA studies is based on distinct structures which can be visualized and epidemiological interpretations of the effects. We developed a qualitative tool that can rapidly screen specific interactions, essential or removable, and a likelihood score that quantifies the extent of the effect. We have identified empirical regularities in applying the proposed method to six GWA data sets with number of SNPs ranged from 100,000 to $1,000,000$.

Under the simplest scenario of two SNPs (SNP1 and SNP2), each having two genotypic variants denoted by 0 and 1 , respectively, we derived the necessary and sufficient conditions to use as a screening tool to quickly and reliably identify pair interactions as essential or removable. We then derived the EI-to-RI likelihood ratio (EI-RI score) to quantify the effects. In investigation of six existing GWA studies we find a consistent pattern of the EI-RI score distributions. Extension to scenarios with SNPs having three genotypes is straightforward, albeit involving tedious calculations.

\section{METHODS}

\subsection{Essential/removable interactions}

The joint risks of a pair of dichotomous markers can be graphically represented by two lines and are depicted in Fig. 1. We use the $O R$ and $\log (O R)$ risk scales; however the results presented can be generalized to any risk scale. Figure 1 illustrates that all two-SNP combinations can be classified into one of three mutually-exclusive categories:

Absolute non-interaction (ANI) (Fig. 1a): No interaction between SNP1 and SNP2 means that the effect of SNP1+SNP2 is the sum of the individual effects (i.e., effects are additive). ANI is manifest by a two-SNP combination 
(a) Absolute non-Interaction

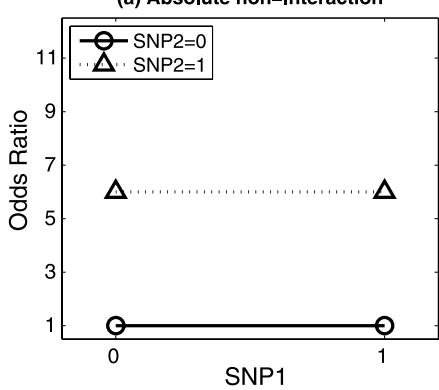

(d) Essential Interaction I: SLOPE

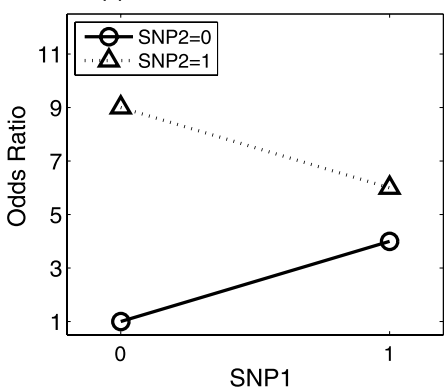

(b) Removable Interaction

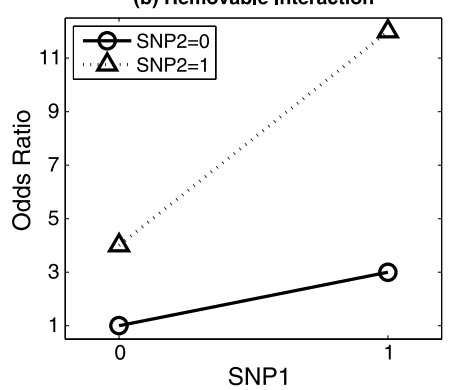

(e) Essential Interaction I: SLOPE

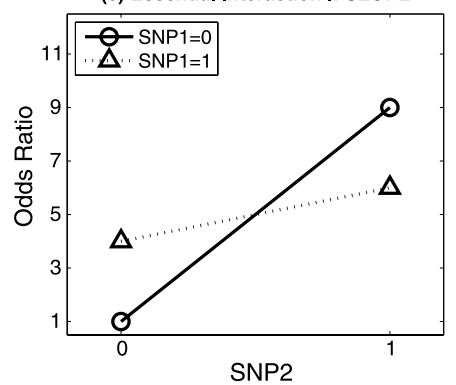

(c) Interaction Removed
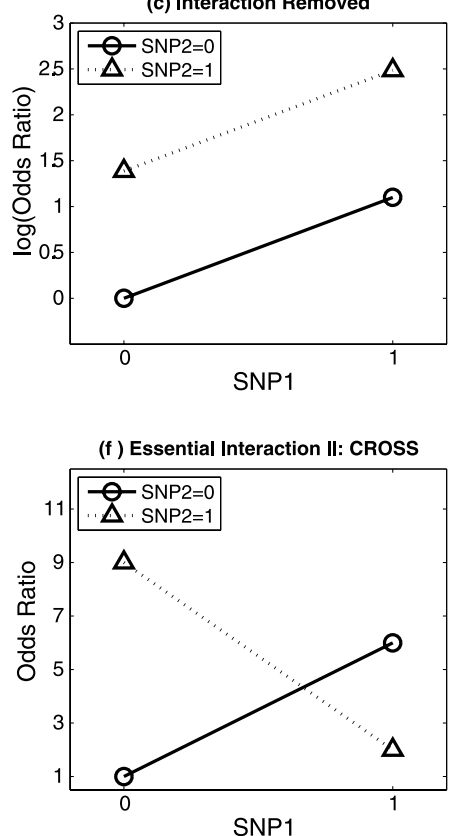

Figure 1. Graphical representations of absolute non-interaction, removable interaction, and essential interaction. (a) Absolute non-interaction (ANI): in the presence of a given genotypic variant of SNP2, risk does not vary by genotypic variant of SNP1

(two lines are parallel with zero slope). This pattern represents ANI because no monotone transformation can force the two lines to not be parallel. (b) There is an interaction (non-intersecting, non-parallel lines with slopes of the same direction) between two SNPs on the OR risk scale, and (c) the interaction is removed (parallel lines) by transformation to the $\log (O R)$ scale. In this instance, a monotone transformation made the two lines parallel. (b) and (c) correspond to the example illustrated in Table 1. (d) and (e) The same essential interaction (EI SLOPE) plotted in two different ways, (d) with SNP1 represented on the $x$-axis, in which the two lines have slopes of opposite directions but do not intersect and (e) with SNP2 represented on the $x$ axis, in which the two lines have slopes of the same direction, and intersect. The patterns shown in (d)

and (e) are equivalent. (f) Essential interaction $\mathrm{EI}_{\mathrm{CROSS}}$, in which the two lines have slopes of opposite direction and intersect. (d), (e), and (f) represent Els because no monotone transformation can make the two lines parallel under any of these conditions.

that does not produce an interaction under any risk scale. This phenomenon occurs when in the presence of a given genotypic variant of SNP2, risk does not vary by genotypic variant of SNP1.

Removable interaction (RI), Fig. $1 \mathrm{~b}-\mathrm{c}$, is manifest by a two-SNP combination that produces an interaction under at least one risk scale (Fig. 1b), and does not produce an interaction under at least one other risk scale (Fig. 1c). If an RI exists, the direction, positive or negative, of the risk difference for one SNP is not affected by the other SNP (and is not zero). However, under a risk scale in which the RI manifests itself as an interaction, the magnitude of the effect of SNP1 varies by SNP2 genotypic stratum, and vice versa. That is, with an RI, the magnitude, but not the direction, of the effect for one SNP may be modified by the other SNP. An example of an RI is given in Table 1.

Table 1. An example of a removable interaction (RI). The interaction between two SNPs (SNP1 and SNP2) is significant under the odds ratio (OR) scale, but is removed under the $\log (O R)$ scale. This example is graphically illustrated in Fig. 1b-c

\begin{tabular}{|c|c|c|c|c|c|}
\hline SNP1 & SNP2 & \# of cases & \# of controls & Odds ratio(OR) & $\log (\mathrm{OR})$ \\
\hline 0 & 0 & 100 & 100 & 1.0 & 0.0 \\
\hline 0 & 1 & 300 & 100 & 3.0 & 1.099 \\
\hline 1 & 0 & 400 & 100 & 4.0 & 1.386 \\
\hline 1 & 1 & 1200 & 100 & 12.0 & 2.485 \\
\hline \multicolumn{4}{|c|}{ Expected risk of " 11 " with no interaction } & 6.0 & 2.485 \\
\hline \multicolumn{4}{|c|}{ Estimation of interaction } & 6.0 & 0.0 \\
\hline \multicolumn{4}{|c|}{$F$-Test of interaction } & $\mathrm{P}=0.0001$ & $\mathrm{P}=1.0$ \\
\hline
\end{tabular}

162 C. Wu et al. 
Essential interaction (EI), Fig. 1d-f, is manifest by a twoSNP combination that produces an interaction under all risk scales: In contrast to RI, an EI exists when the direction of the effect of one SNP is dependent on the genotypic variant of the other SNP. That is, the effect of one SNP is reversed by the other SNP. And as in RI, for an EI the magnitude of the effect for one SNP may also be modified by the other SNP. EI can be further classified into two subclasses, $\mathrm{EI}_{\mathrm{SLOPE}}$ and $\mathrm{EI}_{\mathrm{CROSS}}$. $\mathrm{EI}_{\mathrm{SLOPE}}$ occurs when the reversal of the direction of effect is one way (e.g., SNP1 by SNP2 but

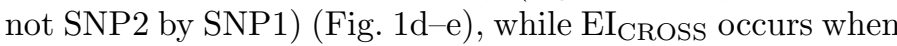
the reversal of the direction of effect is reciprocal (SNP1 by SNP2 and SNP2 by SNP1) (Fig. 1f).

From the above reasoning we can derive a set of relationships in terms of magnitudes and directions of the effect change of one SNP by the other:

Condition $I$ (slopes have opposite direction): $\left(O R_{10}\right.$ $\left.O R_{00}\right)\left(O R_{11}-O R_{01}\right) \leq 0$ in which one risk difference in the product can equal zero, but not both.

Condition II (lines intersect): $\left(O R_{01}-O R_{00}\right)\left(O R_{11}-\right.$ $\left.O R_{10}\right) \leq 0$ in which one risk difference, but not both, can equal zero.

An interaction is essential if and only if Condition $I$ and/or II holds. If only one of these conditions holds, we have an $\mathrm{EI}_{\mathrm{SLOPE}}$; if both conditions hold, we have an $\mathrm{EI}_{\mathrm{CROSS}}$. If both conditions are not satisfied, then we have RI (unless both product terms in Condition I or Condition $I I$ are zeros, in which case we have ANI).

\subsection{An EI-RI score: a quantitative measure for the effect}

Since the Conditions $I-I I$ are invariant under any monotone transformation, $O R_{a b}$ in two conditions could be replaced by its logarithm, the log odds ratio of genotypic variants combination $a b$ with respect to baseline 00. Based on the Condition $I$ and/or Condition II, the necessary and sufficient(N\&S) condition for EIs, a statistical test can be constructed for the null hypothesis that no EI exists. By replacing $O R$ s with their logarithms, the testing hypothesis depends only on log-odds ratios. The standard theory $[11,13,14]$ assures that the likelihood ratio test for such kind of hypothesis based on the prospective likelihood function would be valid. The prospective likelihood function is proportional to

$$
L(\theta)=\prod_{a=0}^{1} \prod_{b=0}^{1} p_{a b}^{n_{a b}}\left(1-p_{a b}\right)^{m_{a b}}
$$

where $n_{a b}$ and $m_{a b}$ respectively are observed numbers of cases and controls for joint genotypic variant $a b, p_{a b}=$ $e^{\theta_{a b}} /\left(1+e^{\theta_{a b}}\right)$ is the corresponding affected probability and $\theta_{a b}$ represents the $\log$ odd of the joint genotypic variant $a b$. Let $\theta=\left(\theta_{00}, \theta_{01}, \theta_{10}, \theta_{11}\right)$ be the vector of parameters. The null hypothesis parameter space, the complement of the subset for parameters satisfying the N\&S condition, is not closed. We will use instead its closure, i.e.,

$$
\begin{gathered}
\Theta_{0}=\left\{\theta:\left(\theta_{01}-\theta_{00}\right)\left(\theta_{11}-\theta_{10}\right) \geq 0\right. \text { and } \\
\left.\left(\theta_{10}-\theta_{00}\right)\left(\theta_{11}-\theta_{01}\right) \geq 0\right\}
\end{gathered}
$$

as a working null parameter space. By doing so, we can simplify the computation and the test will be slightly conservative.

We took the EI-RI score to be the likelihood ratio statistics [7], i.e.

$$
2\left(\max _{\theta \in \Theta} \log L(\theta)-\max _{\theta \in \Theta_{0}} \log L(\theta)\right)
$$

where $\Theta=\left\{\theta:-\infty \leq \theta_{00}, \theta_{01}, \theta_{10}, \theta_{11}<\infty\right\}$ is the unconstrained parameter space.

Obviously, the unconstrained $\operatorname{MLE}(\theta \in \Theta)$ has a closed form, namely $\theta_{a b}=\log \left(n_{a b} / m_{a b}\right)$. To obtain the EI-RI score, one needs to calculate the constrained MLE under the null hypothesis, which can be solved by a non-linear programming (NLP) algorithm. However, the NLP algorithm is timeconsuming and could break down especially when the constrained MLE is on the boundary of $\Theta_{0}$. Therefore, the NLP algorithm is not suitable for GWA study.

By virtue of the concavity of the log likelihood function, we developed an efficient algorithm for calculating the constrained MLE. Details of the algorithm are given in Appendix A.

\subsection{EI-RI scores in six GWA data sets}

Under the null hypothesis of ANI or RI, the null distribution of the EI-RI score is mathematically intractable as it is a mixture of zero (when the maximum point belongs to the null parameter space) and a positive distribution (when the constrained MLE does not belong to the null parameter space) with the component weights unknown. Estimation of p-values by the permutation test fails because the conventional permutation procedure is not suitable for our composite null hypothesis. In fact, randomly allocating the samples under the assumption of no joint effects or marginal effects as in the conventional permutation approach inevitably introduces false positives. And indeed, serious inflated type I error rates by the permutation test have been observed in our simulation studies (data not shown here).

Re-sampling approaches [4] might be applicable for estimating p-values with small numbers of SNP markers, but it is beyond computational capability for GWA data with large numbers of SNP pairs. We therefore sought to examine the empirical distribution patterns of the scores among six GWA data sets. All empirical distributions of EI-RI scores in six data sets give rise to similar upper tails, which, in turn, may justify the existence of a universal threshold for declaring the significance of an EI. 
Table 2. Descriptive information of six GWA data sets

\begin{tabular}{|c|c|c|c|c|c|c|}
\hline & GWA1_100K & GWA2_100K & GWA3_300K & GWA4_300K & GWA5_300K & GWA6_1.8m \\
\hline \multicolumn{7}{|l|}{ Sample information } \\
\hline Number of samples & 500 & 441 & 541 & 183 & 268 & 292 \\
\hline Number of cases & 354 & 313 & 270 & 92 & 176 & 175 \\
\hline Number of controls & 146 & 128 & 271 & 91 & 92 & 117 \\
\hline \multicolumn{7}{|l|}{ SNP information } \\
\hline Number of SNPs & 105834 & 109924 & 317503 & 317503 & 317503 & 909622 \\
\hline Number of SNPS on sex chromosome & 0 & 0 & 9173 & 9173 & 9173 & 37380 \\
\hline SNPs with call rate less than $95 \%$ & 1584 & 1018 & 3101 & 7235 & 28124 & 131770 \\
\hline $\begin{array}{l}\text { SNPs with minor allele frequency (MAF) } \\
<0.01\end{array}$ & 5005 & 10642 & 252 & 30970 & 45173 & 39408 \\
\hline SNPs with no polymorphism observed & 1810 & 2150 & 91 & 20540 & 31852 & 66 \\
\hline SNPs with only heterozygotes observed & 0 & 2 & 0 & 0 & 113 & 2046 \\
\hline $\begin{array}{l}\text { Polymorphic SNPs with no heterozygote } \\
\text { observed }\end{array}$ & 25 & 94 & 5 & 139 & 358 & 130957 \\
\hline SNPs with HWE $\chi^{2}>50$ in case group & 26 & 205 & 9126 & 396 & 10084 & 6511 \\
\hline SNPs with HWE $\chi^{2}>50$ in control group & 330 & 666 & 7741 & 596 & 2117 & 4555 \\
\hline $\begin{array}{l}\text { SNPs with HWE } \chi^{2}>50 \text { in combined } \\
\text { samples }\end{array}$ & 752 & 1112 & 9265 & 5443 & 12160 & 10023 \\
\hline \multicolumn{7}{|l|}{ SNP QC Results Summary: } \\
\hline Number of autosomal chromosome Markers & 105834 & 109924 & 308330 & 308330 & 308330 & 872242 \\
\hline $\begin{array}{l}\text { Autosomal SNPs Passing Call Rate/MAF } \\
\text { Threshold } \\
(0.01 \leq \mathrm{MAF}<0.05 \text { and call rate } \geq 0.99) \\
\text { or }(0.05 \leq \mathrm{MAF}<0.10 \text { and call rate } 0.97) \\
\text { or }(0.10 \leq \mathrm{MAF} \text { and call rate } \geq 0.95)\end{array}$ & 100610 & 96260 & 304342 & 269795 & 256811 & 606999 \\
\hline Pass above, no polymorphism observed & 0 & 0 & 0 & 0 & 0 & 0 \\
\hline Pass above, only heterozygotes observed & 0 & 2 & 0 & 0 & 0 & 6 \\
\hline Pass above, no heterozygote observed & 0 & 0 & 0 & 2 & 100 & 0 \\
\hline $\begin{array}{l}\text { Pass above, fail HWE test }\left(\chi^{2}>50 \text { in case }\right. \\
\text { group or } \chi^{2}>50 \text { in control group) }\end{array}$ & 76 & 191 & 8 & 28 & 3443 & 1303 \\
\hline Number of usable SNPs & 100534 & 96067 & 304334 & 269765 & 253268 & 605690 \\
\hline
\end{tabular}

\section{RESULTS FOR EMPIRICAL STUDY}

Descriptive statistics of six GWA data sets are given in Table 2. The numbers of SNPs in these studies ranged from $10^{5}$ to $10^{6}$. We dichotomized each SNP genotype based on the empirical mode of inheritance [9]. In the six data sets examined, about half ( $48.83 \%$ on average) of the SNP pairs were in the category of EIs (Fig. 1d-f). Of the EI pairs, about two-thirds $\left(65.97 \%\right.$ on average) were $\mathrm{EI}_{\mathrm{SLOPE}}$ pairs and one-third $(34.03 \%)$ were $\mathrm{EI}_{\text {CROss }}$ pairs.

Histogram plots are drawn for EI-RI scores of the six data sets and are shown in Fig. 2a. Comparing histogram plots of the two EI groups, EI $_{\text {CrOss }}$ pairs tend to have higher EIRI scores than EI SLOPE pairs. Patterns of histogram plots are consistent across six data sets, particularly, when EIRI scores become large. This consistency implies that the empirical distributions of EI pairs in the six data sets have similar tail behavior. This conclusion was confirmed by the consistency of tail quantiles for EI-RI scores, as shown in Fig. 2c. The consistent tail distribution of EI-RI scores in six data sets may justify the existence of a universal critical value for declaring the significance of an EI.
In GWA studies, the critical values are chosen as the (1$p$ )-quantiles of EI-RI scores under the null hypothesis for an extremely small $p$. One way to estimate the extreme quantiles is to inverse the linear interpolation of the empirical cumulative distribution function [10]. We found that the empirical (1-p)-quantiles are consistent across the six data sets for $10^{-8} \leq p \leq 10^{-2}$ (Fig. 2c, Table 3). However, in the upper $10^{-8}$ tail region the quantiles became irregular and unpredictable, which is due to random error of the extreme value distribution of $\mathrm{p}$-values. Therefore, the interpolation method is not suitable for estimation of extreme quantiles. To overcome this problem, we approximated extreme quantiles using much lower quantiles which can be estimated by intermediate order statistics.

For each empirical distribution of the six data sets, the relationship between the $(1-p)$ quantile and $\log (p)$ was approximately linear in the range $10^{-8} \leq p \leq 10^{-2}$ (Fig. 2c). The linearity implies that extreme value indices [5] are zero for all six empirical distributions. The conclusion was confirmed by constructing $95 \%$ confidence intervals of the extreme value index with different numbers of upper order 

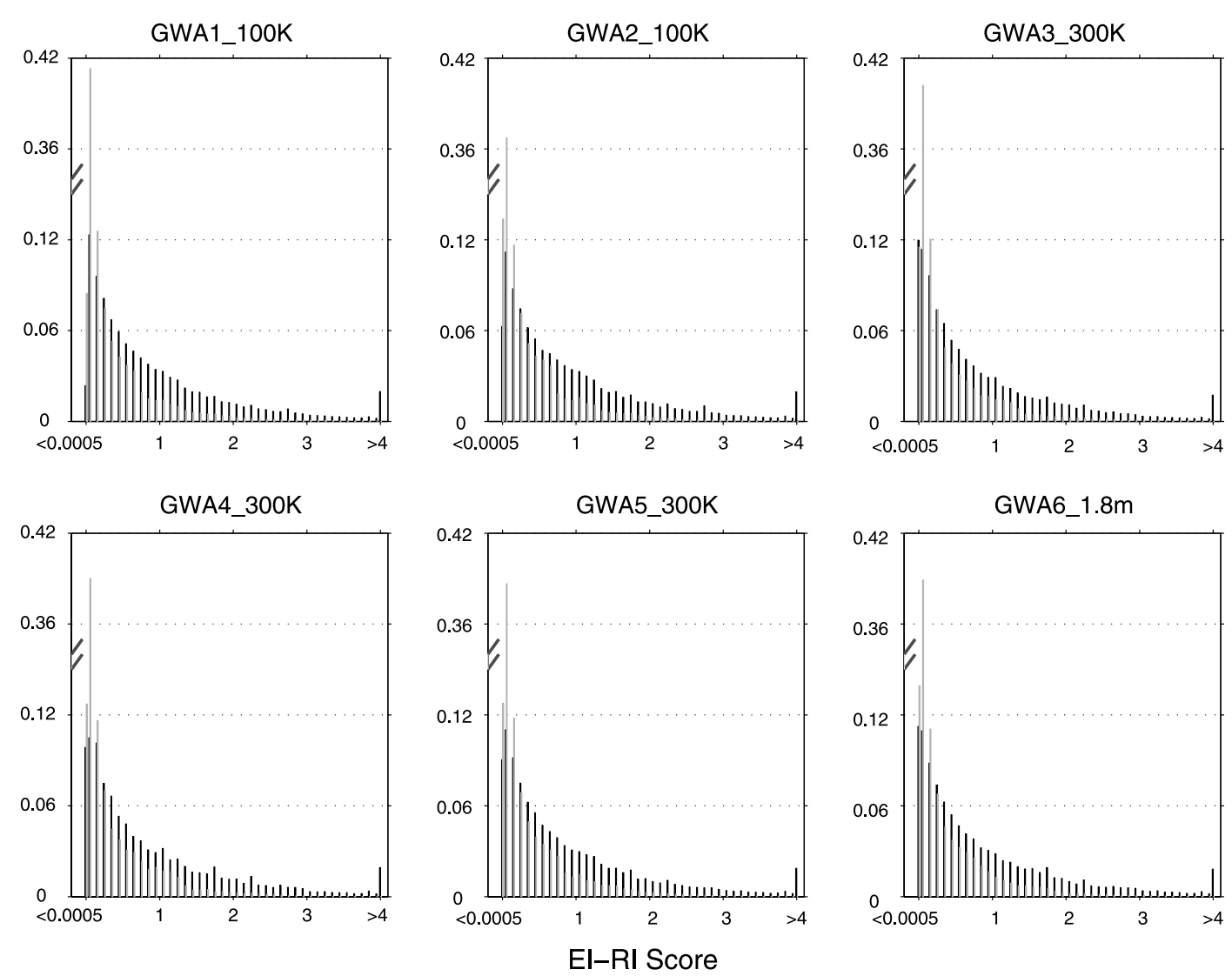

Figure 2a. The histogram plots of El-RI scores in six data sets. Dark black is the histogram for $\mathrm{EI}_{\mathrm{CROSS}}$ pairs and gray is the histogram for $\mathrm{EI}_{\mathrm{SLOPE}}$ pairs. For each type of El pair, the leftmost column is the frequency for El-RI scores less than 0.0005 and the rightmost column is the frequency for El-RI scores larger than 4.

statistics (for details see Appendix B). The value zero belongs to almost all these confidence intervals, which does not contradict the hypothesis that the extreme value index is zero.

With a zero extreme value index, the $(1-p)$-quantile for small $p$ can be approximated by

$$
Q(1-p)=-\sigma \log p+\mu
$$

for some $\mu$ and $\sigma(\sigma>0)$. That is, the upper tail of the EI-RI score approximately follows an exponential distribution with location parameter $\mu$ and scale parameter $\sigma$. The two parameters were estimated by fitting the regression line defined in (4) for $p$ in the range $\left[10^{-8}, 10^{-2}\right]$. As shown in Table 4, all R-square statistics are close to 1 , which indicates that regression lines defined in (4) almost perfectly fit the data (also see quantile-quantile plots in Fig. 2b).

Furthermore, estimators of the two parameters are consistent across all six data sets. This, again, suggests that distributions of EI-RI scores have similar tail behavior.

Based on these data and a reasonable underlying assumption of an exponential tail distribution for EI-RI scores under the null hypothesis, we can deduce a simple proposition: the EI-RI score corresponding to the $(1-p)$-quantile is approximately equal to $(-2.617) \times \log (p)-2.483$, for small $p$, and equivalently, the p-value is approximately equal to $\exp \{-($ EI-RI score +2.483$) / 2.617\}$, for large EI-RI scores.

It will be interesting to see whether a similar trend of the scores will hold and the above estimated p-values can be generalized for any GWA study. If so, this in turn may provide a simple guideline of choosing significance levels for SNP pairs worthy of further investigation such as replication studies. For example, here we postulate an EI-RI score less than 18 indicates an inconclusive interaction; a score between 18 and 26 suggests a possibility of a true EI; and a score greater than 26 indicates a statistically significant EI. These speculations warrant further investigation.

\section{DISCUSSION}

Detection of interaction effects among SNPs represents the next frontier in GWA studies. However, screening the enormous array of SNP combinations represents a daunting task. To begin to address this challenge, we classified all twoSNP combinations into three mutually-exclusive categories, absolute non-interactions, removable interactions, and essential interactions, and we characterized the conditions for 

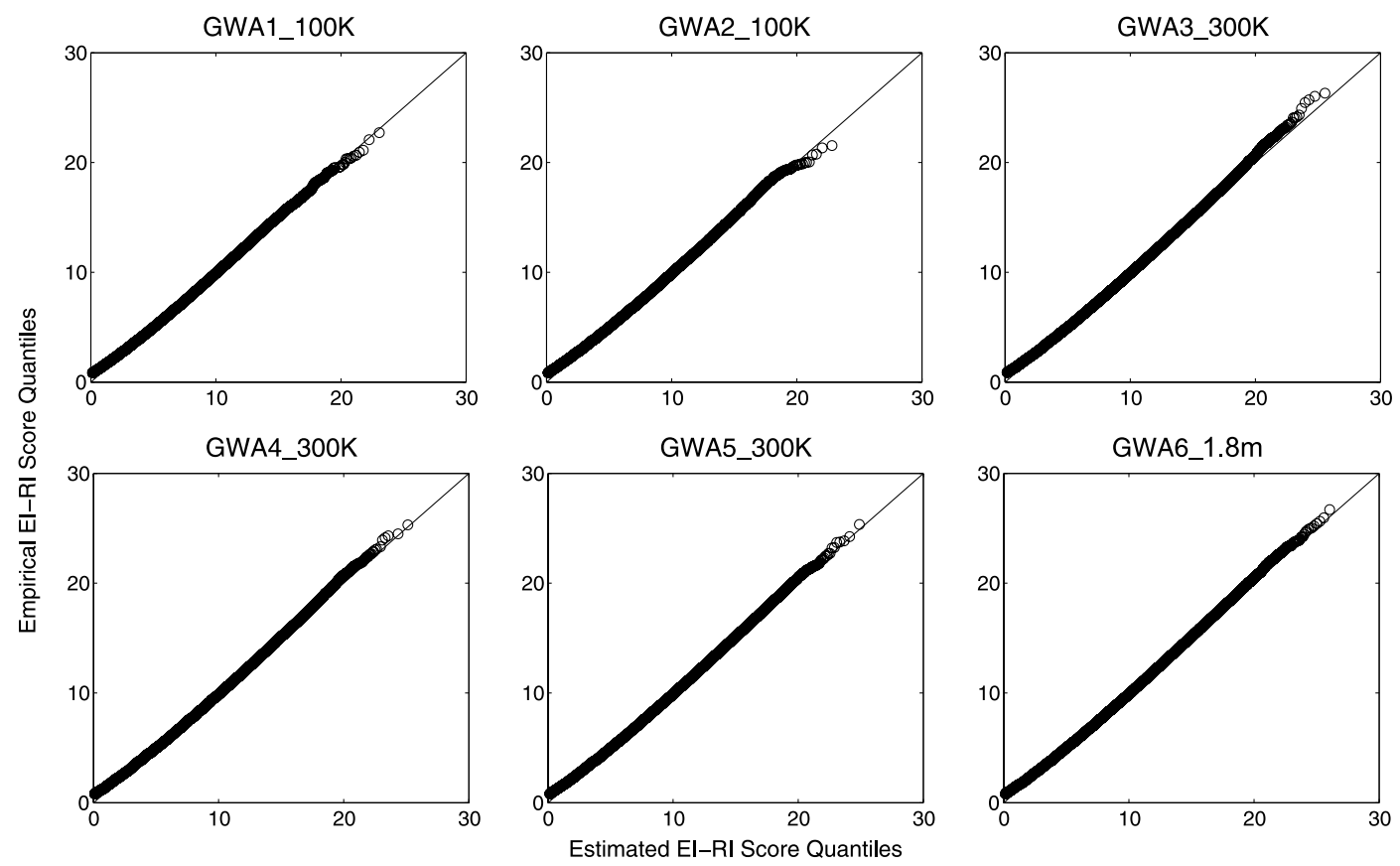

Figure 2b. Quantile-quantile plots of El-RI scores for six GWA data sets. Each point corresponds to a probability p: the $x$-coordinate represents the El-RI score corresponding to the $p$-th quantile of the exponential distribution (details in text) and the $y$-coordinate represents the El-RI score corresponding to the $p$-th sample quantile from the empirical data.

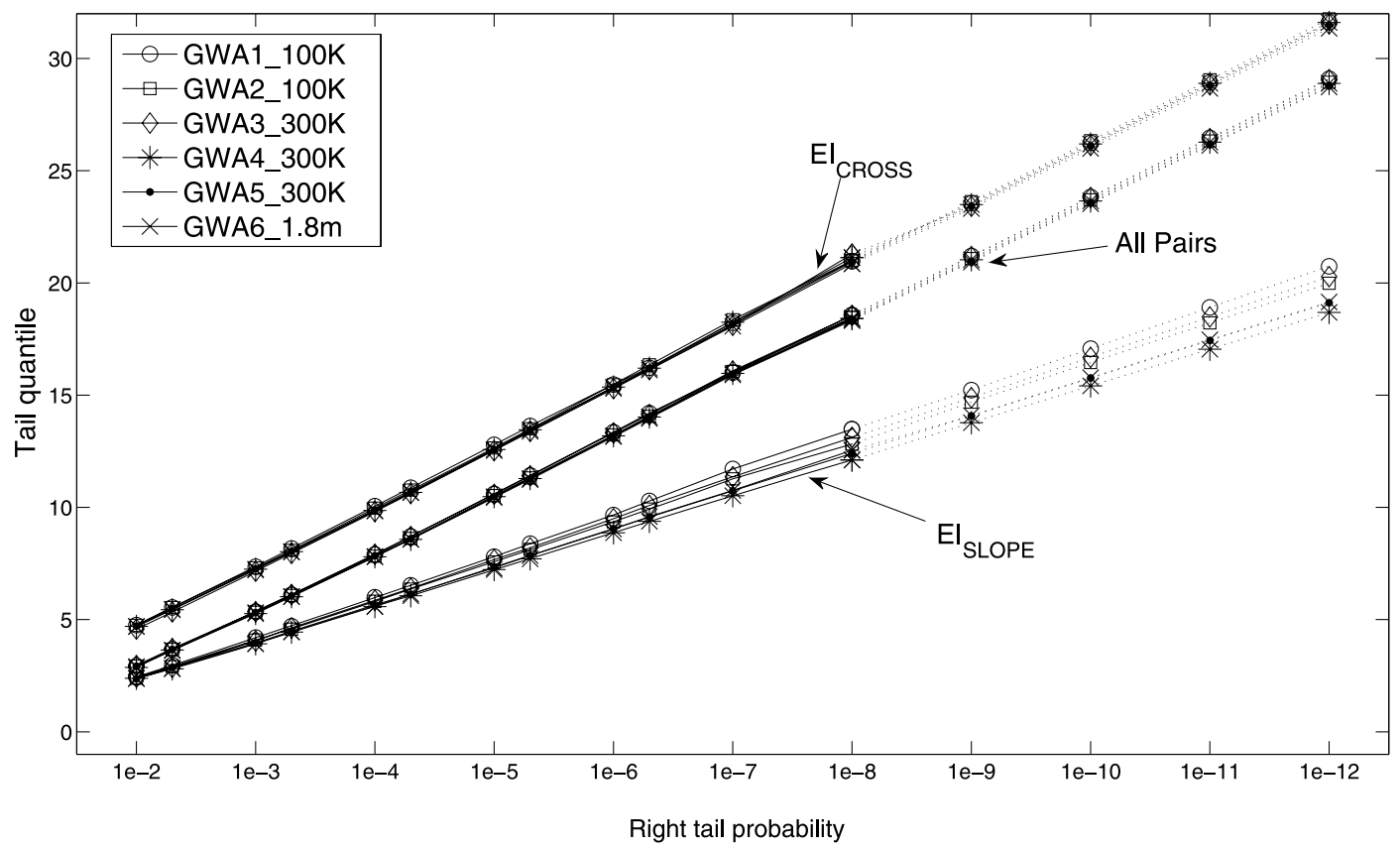

Figure 2c. The tail quantiles of the El-RI scores stratified by $\mathrm{EI}_{\mathrm{CROSS}}$ pairs and $\mathrm{EI}_{\mathrm{SLOPE}}$ pairs in the six GWA data sets based on the real data (solid lines) and the extrapolated tail quantiles from an estimated exponential distribution (dotted lines).

each of these categories. In essence, an interaction is removable when the magnitude, but not the direction, of the effect of one SNP is modified by the other SNP, and an interaction is essential when the direction of the effect for at least one of the SNPs is altered in the presence of the other SNP (and the magnitude may be modified as well).

We developed an efficient computer program to screen for EIs and to calculate the EI-RI scores (likelihood ratio

166 C. Wu et al. 
Table 3. Right tail quantiles of the El scores in the six GWA data sets. Quantiles between $10^{-8}$ and $10^{-2}$ were estimated directly from the data, outside that range were estimated by extrapolation

\begin{tabular}{|c|c|c|c|c|c|c|}
\hline Tail probability & GWA1_100K & GWA2_100K & GWA3_300K & GWA4_300K & GWA5_300K & GWA6_1.8m \\
\hline $1.0 \mathrm{E}-03$ & 5.3524 & 5.3309 & 5.3555 & 5.2672 & 5.3139 & 5.3129 \\
\hline $1.0 \mathrm{E}-04$ & 7.9038 & 7.8264 & 7.9108 & 7.7986 & 7.8368 & 7.8085 \\
\hline $5.0 \mathrm{E}-05$ & 8.7071 & 8.6172 & 8.7007 & 8.5751 & 8.6036 & 8.5792 \\
\hline $1.0 \mathrm{E}-05$ & 10.5998 & 10.5225 & 10.5844 & 10.4760 & 10.4865 & 10.4403 \\
\hline $5.0 \mathrm{E}-07$ & 14.1986 & 14.0553 & 14.1349 & 14.0286 & 14.0091 & 13.9621 \\
\hline $1.0 \mathrm{E}-07$ & 16.0027 & 16.0606 & 16.0798 & 15.9701 & 15.9223 & 15.9039 \\
\hline $1.0 \mathrm{E}-08$ & 18.5659 & 18.4649 & 18.5488 & 18.4146 & 18.3703 & 18.3299 \\
\hline $1.0 \mathrm{E}-09$ & 21.1983 & 21.0851 & 21.1762 & 21.0338 & 20.9728 & 20.9296 \\
\hline $1.0 \mathrm{E}-10$ & 23.830 & 23.7053 & 23.8035 & 23.6531 & 23.5754 & 23.5293 \\
\hline
\end{tabular}

Table 4. Estimation for intercept $\mu$ and slope $\sigma$ by fitting $(1-p)$-empirical quantile vs $-\log (p)$ for $10^{-8} \leq p \leq 10^{-2}$

\begin{tabular}{ccccccc}
\hline & GWA1_100K & GWA2_100K & GWA3_300K & GWA4_300K & GWA5_300K & GWA6_1.8m \\
\hline$\mu$ & -2.493 & -2.492 & -2.475 & -2.519 & -2.450 & -2.468 \\
$\sigma$ & 2.632 & 2.620 & 2.627 & 2.619 & 2.603 & 2.600 \\
R-square & 0.9994 & 0.9991 & 0.9993 & 0.9992 & 0.9992 & 0.9992 \\
\hline
\end{tabular}

statistics) for each SNP pair in GWA data of any number of SNPs. With this algorithm we examined the distributions of EI-RI scores in six GWA data sets with $10^{5}$ to $10^{6}$ SNPs. A consistent pattern emerged, which allowed us to derive the tail quantiles by extrapolation from the stable portion of the empirical distributions. Finally, we suggested an EI-RI score threshold for identifying prime candidate EIs.

The EI-RI score presented here emphasizes EIs because they are not dependent on risk scale and represent the most extreme form of interaction (reversal of direction of risk difference). Furthermore, while one may reasonably screen for RIs solely among SNPs that exhibit significant main effects, this approach may not work for EIs, especially for $\mathrm{EI}_{\mathrm{CROSS}}$. This is because in the presence of an RI, both SNPs have non-zero marginal effects (of course, the magnitude of the effects depends on the joint effects and MAFs). With EI, however, the marginal effect for at least one SNP can be close to zero even when the EI effect is substantial. With $\mathrm{EI}_{\text {CROSS }}$ the marginal effect for both SNPs can be close to zero. Thus, many EIs may be missed if only SNPs with main effects are screened. This conjecture requires further investigation.

Even though RIs have the undesirable mathematical property of dependence on the risk scale, it is likely that many RIs in GWA data sets will prove to be potentially interesting, as they have proved to be in traditional casecontrol studies. It will be straightforward to apply the meth- ods presented here, which are independent of risk scale, to screen for RIs as well as EIs.

Taken together, the qualitative and the quantitative methods we proposed to detect interaction effects in GWA data by way of EIs and RIs is conceptually simple, computationally manageable and, most of all, the resulting interactions can be readily visualized and unequivocally interpreted. We plan to determine the practical utility of our current methodology to address real disease problems.

All algorithms have been implemented in $\mathrm{C}++$ and the program is available upon request from authors.

\section{ACKNOWLEDGEMENTS}

The research was partially supported by NIH/NEI grants (R01 EY015771, R21 EY018127), NIH/NHGRI grant (K25 HG000060), NIH grant (5-R37-GM047845-18), NSF grant (DMS-05-04871), NSFC grants (10701067, 10671189), and grants provided by Verto Institute, American Health Alliance Foundation, Macular Vision Research Foundation, and Ellison Medical Researching Foundation. This work was carried out while Zhang was a postdoctoral associate and Yang was a visiting scholar in Hoh's Lab at Yale. We gratefully acknowledge Yale University Biomedical High Performance Computing Center and NIH grant: RR19895, which funded the instrumentation. Our thanks also extend to the database of Genotypes and Phenotypes (dbGaP) for the 


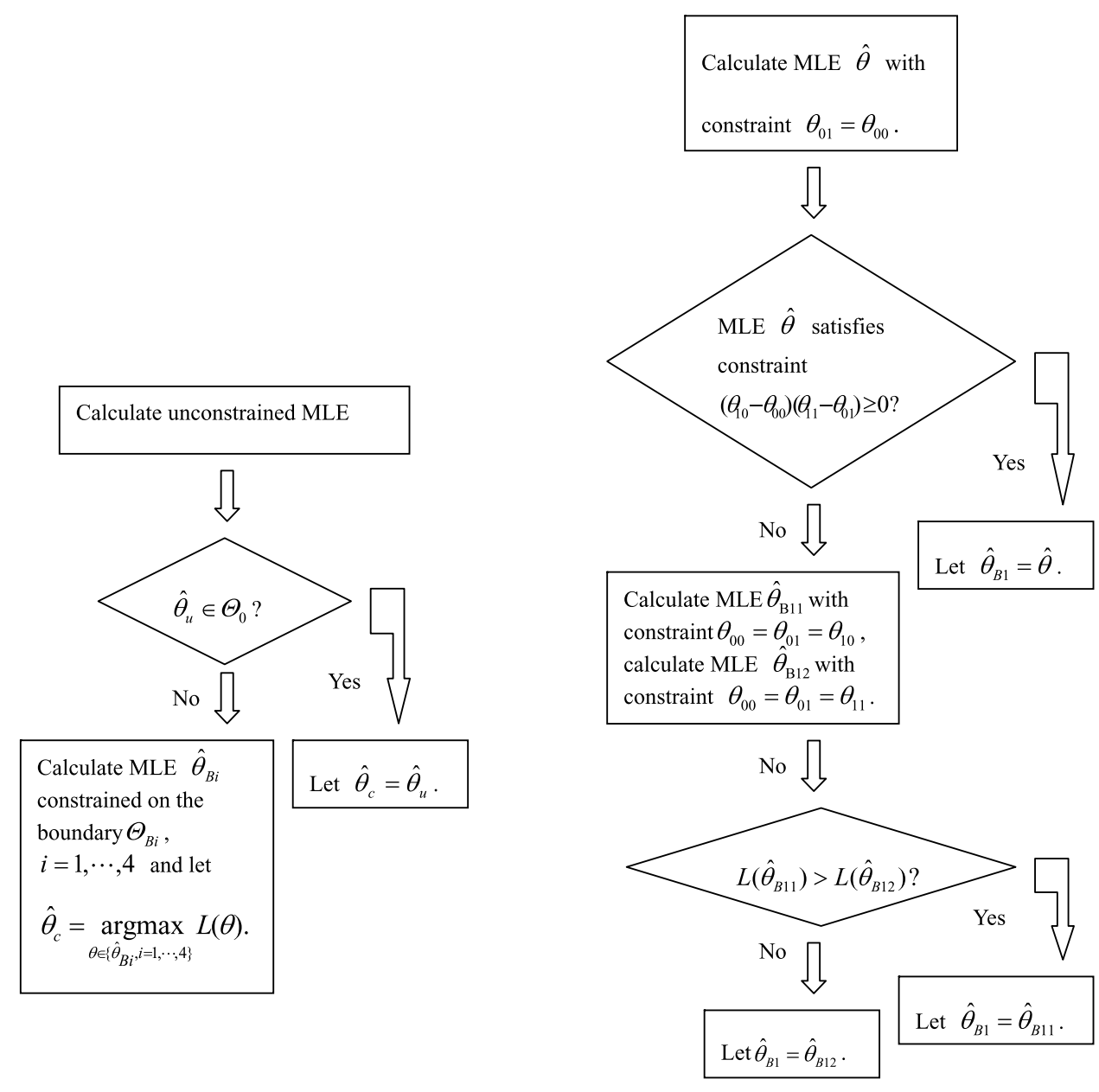

(1)

(2)

Figure A. Algorithm for calculating MLE constrained on the null hypothesis. (1) Calculation of constrained MLE $\hat{\theta}_{c}$. (2) Calculation of constrained MLE $\hat{\theta}_{B 1}$ on boundary $\Theta_{B 1}$.

GWA data sets. The content is solely the responsibility of the authors and does not necessarily represent the official views of the National Institutes of Health. The authors would like to thank the editor for his comments which improved this article.

\section{APPENDIX A. ALGORITHM FOR CONSTRAINED MLE}

If the unconstrained MLE belongs to $\Theta_{0}$, then it is of course the constrained MLE. If the constrained MLE is an interior point of $\Theta_{0}$, it must be the unconstrained MLE since the $\log$ likelihood function is concave so that there are no local maxima other than the global maxima. Therefore, if the unconstrained MLE does not belong to $\Theta_{0}$, the constrained MLE must be on its boundary $\Theta_{B}$. This leads to the algorithm for obtaining the constrained MLE, which is illustrated in Fig. A1.
The boundary $\Theta_{B}$ is the union of four sub-boundaries: $\Theta_{B 1}=\left\{\theta: \theta_{01}=\theta_{00}\right.$ and $\left.\left(\theta_{10}-\theta_{00}\right)\left(\theta_{11}-\theta_{01}\right) \geq 0\right\}$, $\Theta_{B 2}=\left\{\theta: \theta_{10}=\theta_{11}\right.$ and $\left.\left(\theta_{10}-\theta_{00}\right)\left(\theta_{11}-\theta_{01}\right) \geq 0\right\}$, $\Theta_{B 3}=\left\{\theta: \theta_{10}=\theta_{00}\right.$ and $\left.\left(\theta_{01}-\theta_{00}\right)\left(\theta_{11}-\theta_{10}\right) \geq 0\right\}$ and $\Theta_{B 4}=\left\{\theta: \theta_{11}=\theta_{01}\right.$ and $\left.\left(\theta_{01}-\theta_{00}\right)\left(\theta_{11}-\theta_{10}\right) \geq 0\right\}$. We can calculate the MLEs on four sub-boundaries separately and the one with largest likelihood function value is the constrained MLE on boundary $\Theta_{B}$. We also develop an algorithm for obtaining the MLE on sub-boundary $\Theta_{B 1}$ (the other three constrained MLEs can be similarly obtained). The algorithm is graphically displayed in Fig. A2.

The algorithm is very easy to implement and is time efficient since there are closed forms for these constrained MLEs that need to be calculated in the algorithm. For example, under constraint $\theta_{01}=\theta_{00}$, the resulting MLE of $\theta$ is $\left(\log \left[\left(n_{00}+n_{01}\right) /\left(m_{00}+m_{01}\right)\right], \log \left[\left(n_{00}+n_{01}\right) /\left(m_{00}+m_{01}\right)\right]\right.$, $\left.\log \left[n_{10} / m_{10}\right], \log \left[n_{11} / m_{11}\right]\right)$. Under constraint $\theta_{00}=\theta_{01}=$ $\theta_{10}$, MLEs of $\theta_{i j} \mathrm{~s}$ are $\theta_{00}=\theta_{01}=\theta_{10}=\log \left[\left(n_{00}+\right.\right.$ 
Table A. Moment estimator and the 95\% asymptotic confidence interval for extreme value index $c$ with different $k$ 's, where $k$ is the number of upper order statistics used in the moment estimator

\begin{tabular}{|c|c|c|c|c|c|c|c|c|c|}
\hline \multirow[t]{3}{*}{$k$} & \multirow{2}{*}{$\begin{array}{l}\text { Moment } \\
\text { Estimation }\end{array}$} & \multicolumn{2}{|c|}{$\begin{array}{l}95 \% \text { Confidence } \\
\text { Interval }\end{array}$} & \multirow{2}{*}{$\begin{array}{l}\text { Moment } \\
\text { Estimation }\end{array}$} & \multicolumn{2}{|c|}{$\begin{array}{l}95 \% \text { Confidence } \\
\text { Interval }\end{array}$} & \multirow{2}{*}{$\begin{array}{l}\text { Moment } \\
\text { Estimation }\end{array}$} & \multicolumn{2}{|c|}{$\begin{array}{l}95 \% \text { Confidence } \\
\text { Interval }\end{array}$} \\
\hline & & Lower & Upper & & Lower & Upper & & Lower & Upper \\
\hline & \multicolumn{3}{|c|}{ GWA1_100K } & \multicolumn{3}{|c|}{ GWA2_100K } & \multicolumn{3}{|c|}{ GWA3_300K } \\
\hline 50 & -0.0592 & -0.3266 & 0.2082 & -0.035 & -0.3050 & 0.2349 & 0.0789 & -0.1991 & 0.3570 \\
\hline 100 & -0.0570 & -0.2462 & 0.1322 & -0.2421 & -0.4453 & -0.0390 & -0.0275 & -0.2192 & 0.1642 \\
\hline 200 & -0.0503 & -0.1843 & 0.0838 & -0.2524 & -0.3972 & -0.1075 & -0.0150 & -0.1517 & 0.1218 \\
\hline 300 & -0.0420 & -0.1519 & 0.0678 & -0.2184 & -0.3335 & -0.1033 & -0.0825 & -0.1913 & 0.0263 \\
\hline 400 & 0.0186 & -0.0794 & 0.1166 & -0.1098 & -0.2042 & -0.0154 & 0.0072 & -0.0908 & 0.1052 \\
\hline 500 & 0.0542 & -0.0335 & 0.1420 & -0.096 & -0.1803 & -0.0117 & 0.0001 & -0.0875 & 0.0878 \\
\hline 1000 & -0.0044 & -0.0662 & 0.0573 & -0.0296 & -0.0902 & 0.0309 & 0.0129 & -0.0491 & 0.0749 \\
\hline 2000 & 0.0066 & -0.0372 & 0.0504 & -0.0199 & -0.0629 & 0.0232 & 0.0528 & 0.0089 & 0.0967 \\
\hline 3000 & -0.0194 & -0.0546 & 0.0158 & -0.0021 & -0.0378 & 0.0336 & 0.0113 & -0.0245 & 0.0471 \\
\hline 4000 & -0.0391 & -0.0692 & -0.0090 & 0.0341 & 0.0031 & 0.0651 & 0.0396 & 0.0086 & 0.0706 \\
\hline 5000 & -0.0131 & -0.0405 & 0.0143 & -0.0021 & -0.0297 & 0.0256 & 0.0347 & 0.007 & 0.0624 \\
\hline \multirow[t]{2}{*}{10000} & -0.0345 & -0.0536 & -0.0155 & 0.0283 & 0.0087 & 0.0479 & 0.0472 & 0.0276 & 0.0668 \\
\hline & \multicolumn{3}{|c|}{ GWA4_300K } & \multicolumn{3}{|c|}{ GWA5_300K } & \multicolumn{3}{|c|}{ GWA6_1.8m } \\
\hline 50 & -0.0833 & 0.1949 & 0.3614 & -0.0858 & 0.1924 & 0.3640 & 0.0349 & -0.2424 & 0.3123 \\
\hline 100 & 0.013 & -0.1830 & 0.2091 & -0.0255 & -0.2174 & 0.1665 & -0.0255 & -0.2175 & 0.1664 \\
\hline 200 & -0.0827 & -0.2159 & 0.0506 & -0.0939 & -0.2271 & 0.0394 & -0.1262 & -0.2602 & 0.0079 \\
\hline 300 & -0.0561 & -0.1654 & 0.0531 & -0.0531 & -0.1625 & 0.0562 & -0.0848 & -0.1935 & 0.0240 \\
\hline 400 & -0.0568 & -0.1514 & 0.0378 & -0.0383 & -0.1336 & 0.057 & -0.0559 & -0.1505 & 0.0387 \\
\hline 500 & -0.0962 & -0.1805 & -0.0119 & -0.0179 & -0.1042 & 0.0684 & -0.0656 & -0.1500 & 0.0188 \\
\hline 1000 & -0.0352 & -0.0956 & 0.0252 & -0.0218 & -0.0827 & 0.0390 & -0.0107 & -0.0721 & 0.0507 \\
\hline 2000 & -0.002 & -0.0457 & 0.0418 & -0.0061 & -0.0497 & 0.0375 & -0.0217 & -0.0647 & 0.0214 \\
\hline 3000 & 0.0107 & -0.0251 & 0.0465 & -0.0227 & -0.0578 & 0.0124 & 0.0067 & -0.0291 & 0.0425 \\
\hline 4000 & 0.0237 & -0.0073 & 0.0547 & 0.0037 & -0.0273 & 0.0347 & 0.0039 & -0.0271 & 0.0349 \\
\hline 5000 & 0.0208 & -0.0069 & 0.0485 & 0.0208 & -0.0069 & 0.0485 & -0.0008 & -0.0285 & 0.0269 \\
\hline 10000 & 0.0126 & -0.0070 & 0.0322 & 0.0018 & -0.0178 & 0.0214 & 0.0014 & -0.0182 & 0.0210 \\
\hline
\end{tabular}

$\left.\left.n_{01}+n_{10}\right) /\left(m_{00}+m_{01}+m_{10}\right)\right]$ and $\theta_{11}=\log \left[n_{11} / m_{11}\right]$. Similarly, under constraint $\theta_{00}=\theta_{01}=\theta_{11}$, the MLE of $\theta_{00}=\theta_{01}=\theta_{11}$ is $\log \left[\left(n_{00}+n_{01}+n_{11}\right) /\left(m_{00}+m_{01}+m_{11}\right)\right]$ and the MLE of $\theta_{10}$ is $\log \left[n_{10} / m_{10}\right]$.

\section{APPENDIX B. MOMENT ESTIMATION AND CONFIDENCE INTERVAL FOR EXTREME VALUE INDEX}

The key issue in extreme value theory is the estimation of the extreme value index $c$, which governs the tail behavior of a distribution function. The Hill estimator is the most popular estimator which is restricted to the case $c>0$. The moment estimator $\hat{c}_{M}$, an adaptation of the Hill estimator, is a consistent estimator for all real value $c$ 's. Let $X_{1, n} \leq X_{2, n} \leq \cdots \leq X_{n, n}$ be the order statistics for sample $X_{1}, \ldots, X_{n}$, the moment estimator of $c$ is given by

$$
\hat{c}_{M}=M_{1}+1-\frac{1}{2}\left(1-\frac{M_{1}^{2}}{M_{2}}\right)^{-1},
$$

with $M_{j}=\frac{1}{k} \sum_{k=0}^{k-1}\left(\log X_{n-i, n}-\log X_{n-k, n}\right)^{j}$ for $j=1,2$.
Under some regular conditions, $\sqrt{k}\left(\hat{c}_{M}-c\right)$ asymptotically follows a zero mean normal distribution with variance [3]

$$
\begin{cases}1+c^{2}, & \text { if } c \geq 0 \\ \frac{(1-c)^{2}(1-2 c)\left(1-c+6 c^{2}\right)}{(1-3 c)(1-4 c)}, & \text { if } c<0\end{cases}
$$

An approximate $(1-\alpha) 100 \%$ confidence interval is then given by

$$
\hat{c}_{M}-Z_{\alpha / 2} \sqrt{\frac{\operatorname{var}\left(\hat{c}_{M}\right)}{k}}<c<\hat{c}_{M}+Z_{\alpha / 2} \sqrt{\frac{\operatorname{var}\left(\hat{c}_{M}\right)}{k}}
$$

where $\operatorname{var}\left(\hat{c}_{M}\right)$ is the asymptotic variance with $c$ being replaced by its moment estimator, and $Z_{\alpha / 2}$ is the $(1-\alpha / 2)$ quantile of the standard normal distribution.

Table A gives the $95 \%$ asymptotic confidence intervals for various values of $k$. The value zero belongs to almost all these confidence intervals, which does not contradict the hypothesis that the extreme value index is zero.

Received 11 April 2009 


\section{REFERENCES}

[1] Breslow, N. E. and DAy, N. E. (1980). Statistical Methods in Cancer Research. Volume I - The Analysis of Case-Control Studies, Lyon, France: IARC Sci. Publ.

[2] Chen, X., Liu, C. T., Zhang, M., and Zhang, H. (2007). A forestbased approach to identifying gene and gene gene interactions. Proc Natl Acad Sci 104 19199-19203.

[3] Dekkers, A. L. M., Einmahl, J. H. J., and Haan, L. D. (1989). A moment estimator for the index of an extreme-value distribution. The Annals of Statistics 17 1833-1855. MR1026315

[4] Efron, B. (1982). The Jackknife, the Bootstrap and Other Resampling Plans, Philadelphia: SIAM. MR0659849

[5] HaAn, L. D. and Ferreira, A. (2006). Extreme Value Theory: An Introduction, New York, London: Springer. MR2234156

[6] Hindorff, L., Junkins, H., and Manolio, T. (2009). A Catalog of Published Genome-Wide Association Studies. Available at: www.genome.gov/26525384.

[7] Lehmann, E. L. and Romano, J. P. (2005). Testing Statistical Hypotheses, New York: Springer. MR2135927

[8] Marchini, J., Donnelly, P., and Cardon, L. R. (2005). Genome-wide strategies for detecting multiple loci that influence complex diseases. Nat Genet 37 413-417.

[9] Matthews, A. G., Haynes, C., Liu, C., and Otт, J. (2008). Collapsing SNP genotypes in case-control genome-wide association studies increases the type I error rate and power. Stat Appl Genet Mol Biol 7:Article23.

[10] Parzen, E. (1979). Nonparametric statistical data modeling. Journal of the American Statistical Association 74 105-121. MR0529528

[11] Prentice, R. L. and Pyke, R. (1979). Logistic disease incidence models and case-control studies. Biometrika 66 403-411. MR0556730

[12] Scheffe, H. (1959). The Analysis of Variance, New York: John Wiley \& Sons MR0116429

[13] Scott, A. J. and Wild, C. J. (1989). Hypothesis testing in casecontrol studies. Biometrika 76 806-808. MR1041428

[14] Seaman, S. R. and Richardson, S. (2004). Equivalence of prospective and retrospective models in the Bayesian analysis of case-control studies. Biometrika 91 15-25. MR2050457

\section{Chengqing $\mathrm{Wu}$}

Yale School of Public Health

New Haven, CT

E-mail address: chengqing.wu@yale.edu
Hong Zhang

Department of Statistics and Finance

University of Science and Technology of China

Hefei, Anhui, P. R. China

E-mail address: zhangh@ustc.edu.cn

Xiangtao Liu

Department of Applied Mathematics

Yale University

New Haven, CT

E-mail address: xiangtao.liu@yale.edu

Andrew DeWan

Yale School of Public Health

New Haven, CT

E-mail address: andrew.dewan@yale.edu

Robert Dubrow

Yale School of Public Health

New Haven, CT

E-mail address: robert.dubrow@yale.edu

\section{Zhiliang Ying}

Department of Statistics

Columbia University

New York, NY

E-mail address: zying@stat.columbia.edu

\section{Yaning Yang}

Department of Statistics and Finance

University of Science and Technology of China

Hefei, Anhui, P. R. China

E-mail address: ynyang@ustc.edu.cn

Josephine Hoh

Yale School of Public Health

New Haven, CT

E-mail address: josephine.hoh@yale.edu 